\title{
The Influence of Asphaltene-Resin Molecular Interactions on the Colloidal Stability of Crude Oil
}

\author{
Masoumeh Mousavi, ${ }^{\mathrm{a}}$ Tahereh Abdollahi, ${ }^{\mathrm{b}}$ Farideh Pahlavan, ${ }^{\mathrm{c}}$ and Elham H. Fini ${ }^{\mathrm{d}}$
}

\section{Masoumeh Mousavi, Ph.D.}

Center for Innovation in Materials, Methods and Management

North Carolina A\&T State University

1601 E. Market St, Greensboro, North Carolina, 27411

E-mail: fereshteh_mou@yahoo.ca

Office phone: $336-285-3676$

Tahereh Abdollahi, Ph.D. Candidate

Department of Physical Chemistry

University of Mazandaran

Mazandaran, IRAN

E-mail: tahere_abdollahi@yahoo.com

\section{Farideh Pahlavan, Ph.D.}

Research Associate

Center for Innovation in Materials, Methods and Management North Carolina A\&T State University

1601 E. Market St, Greensboro, North Carolina, 27411

E-mail: farideh.pahlavan64@ymail.com

Office phone: 336-285-3676

Elham H. Fini, Ph.D., P.E.

Department of Civil Engineering

North Carolina A\&T State University

1601 E. Market St, Greensboro, North Carolina, 27411

Email: efini@ncat.edu

Office phone: 336-285-3676 


\section{The Influence of Asphaltene-Resin Molecular Interactions on the Colloidal Stability of Crude Oil}

\section{Abstract}

In the colloidal hypothesis of vacuum residua and asphalt, micelles are assumed to have an asphaltenic core with the greatest weight and aromaticity and an outer shell composed of lighter and less aromatic molecules. The true nature of the micelles, and the asphaltenic core in particular, has always been a debatable issue among asphalt researchers. One of the frequently asked questions is whether asphaltenes exist as stacked layers within the micelles or as single molecular units stabilized by resin molecules. In this paper, we investigate the correlation between the number of asphaltene sheets and the final stability of the system in a medium of asphaltene and resin components. Our evaluation is based on rigorous quantum mechanical calculations using a DFT-D approach. Our quantitative findings corroborate the view that the colloidal behavior of crude oil is better described by asphaltene-asphaltene associations. Moreover, our calculations show that if both asphaltene and resin are present, resin-asphaltene interactions are preferred over asphaltene-asphaltene interactions only if the number of resin molecules per micelle is greater than the number of asphaltene molecules per micelle.

Keywords: colloidal state, asphaltene micelles, petroleum resin, density functional theory (DFT) (1)

65

6




\section{Introduction}

The bitumen refining process uses a distillation column or fractionation tower to separate lighter petrochemical fractions from the non-boiling components, which are known as an atmospheric residue. The atmospheric residue, a dark and viscous adhesive material, is then introduced to a vacuum distillation unit to be prepared for the solvent de-asphalting process. Asphaltenes and maltenes are two main fractions of the extracted asphalt. Asphaltene is the wax-free material that precipitates in light paraffinic solvents, while resins along with saturates and aromatics constitute the maltene phase of asphalt. ${ }^{[1],[2]}$ Although characterization of asphaltene and resin has been a controversial topic among the research community, there seems to be a commonly accepted view that these components, as the heaviest, most polar, and most aromatic components of crude oil, contain polycyclic aromatic sheets with a variety of functional groups, some traces of transition metal elements, and alkyl chains on the edges.

The matter of asphaltene precipitation and its technical consequences in refinery processing is a serious concern in the petroleum industry. Self-association of the asphaltene sheets is considered as the first step in the formation of precipitated asphaltene particles. The nature of the stacks formed (number, orientation angle, and interlayer spacing between asphaltene monomers) has been frequently investigated in asphaltene studies. Based on high-resolution transmission electron microscopy (HRTEM) and fluorescence depolarization techniques, the size of asphaltene stacks is estimated to be about $1 \mathrm{~nm}$ (width) $\times$ $1 \mathrm{~nm}$ (height), mainly comprised of two or three individual asphaltene molecules. ${ }^{[3],[4],[5],[6],[7]}$ However, new findings of laboratory centrifugation of a live crude oil indicate that the size of asphaltenes in crude oil is $\sim 2 \mathrm{~nm}$, and their aggregation number (in nano-aggregates) is between $3-8 .{ }^{[8]}$

The micellar model is one of the multiple models proposed to describe asphaltene behavior in crude oil. Despite its limitations, ${ }^{[9],[10],[11]}$ this model has been generally accepted ${ }^{[12],[13],[14],[15],[16],[17]}$ and is the most widely used viewpoint in descriptions of the nature of vacuum residua (VR) and bitumen. The idea of colloidal suspension of asphaltenes in bitumen/crude oil goes back to experiments by Nellensteyn ${ }^{[18]}$ in 1924 that were further developed by Pfeiffer and Saal in later years. ${ }^{[19],[20]}$ Since then, outstanding contributions have been made to demonstrating the colloidal structure of vacuum residua and the role of petroleum resins in the micellar model. ${ }^{[13],[14],[21],[22],[23],[24],[25],[26],[27]}$ These micelles are molecular aggregates of heavy 
asphaltene molecules that are peptized by light resin molecules, dispersing in a surrounding medium of lighter resins, aromatics, and saturates. ${ }^{[28]}$ Resin molecules are well known for their stabilizing effect on asphaltene molecules and suspending them in a medium. It should be noted that the micellar model of asphaltene, in its traditional definition of one micelle, has been recently criticized by some research groups ${ }^{[29],[30],[31]}$ who believe that resin molecules do not act as "surfactants" and strongly question the stabilizing or peptizing effect of resins on asphaltene aggregates, though they accept the general hypothesis of colloidal dispersion of asphaltene in crude oil/bitumen/organic solvents. In the current study, irrespective of the role of resin molecules, the term of "micelle" is used for a nano-aggregate structure composed of an asphaltenic core surrounded by lighter resins, other aromatic hydrocarbons, and saturates.

There seems to be some evidence that asphaltenes may not form large aggregates in crude oil, rather they exist at a much smaller scale (even a molecular scale) associated with resins in a colloidal structure of crude oil. ${ }^{[14],[32],[33],[34]}$ This view is further strengthened by the fact that asphaltenes with low aggregation number are well dispersed at very low concentrations. ${ }^{[35],[36],[37]}$ Proponents of this view argue on the grounds that asphaltene aggregates are formed only when the phase separation is occurring and asphaltene flocculation/precipitation is starting.

Against this view, other groups of the asphalt community (with a dominant majority) believe that the micellar behavior of crude oil is better described by "aggregates", ${ }^{21],[38],[7]}$ Indeed, they theorize that the asphaltene-asphaltene association builds graphite-like stacks constituting the center of micelles, at which the shape, size, and number of aggregation are functions of the solvent, the temperature, and other environmental constraints. ${ }^{[29]}$ Advocates of this view point out the asphaltene stacks exist not only in solid phase, but also before reaching the critical micelle concentration (CMC) limit that is considered as the onset of precipitation/flocculation. ${ }^{[38]}$

Jefferson Creek ${ }^{[39]}$ describes the nature of studies carried out in this fieldwork; at one end of the spectrum are experimental studies that are involved in compositional analysis of the live single-phase reservoir samples, and at the opposite extreme are computational modeling studies that focus on the isolated molecules in a matrix even quite different from the original crude oil. Nevertheless, all of what we know about asphaltene micellization and its peripheral issues are apparently coming from experimental studies, 
and this approach has shaped our understanding of the issue. Indeed, the contribution of theoretical studies on the complex behavior of the systems like asphaltene under extreme conditions of temperature and pressure is insignificant, and is mostly restricted to classical molecular mechanics/dynamics $(\mathrm{MM} / \mathrm{MD}) .{ }^{[12],[40],[41],[42],}$

In the realm of quantum studies, factors like the specific nature of interactions, the complexity, and the massiveness of asphaltene aggregates/micelles have turned the subject of petroleum micelles into a challenge from a computing resource perspective. Additionally, the diversity of interpretations and the lack of consensus on experimental results (structural information, in particular) make asphaltene modeling even more challenging. Accordingly, this challenge and some doubts about the true nature of asphaltene micelles were the main motivation for the present work. In this work, we investigate the interplay between asphaltene and resin molecules in the formation of petroleum micelles. We also try to find a solution for the frequently posed question of whether individual petroleum micelles are stacked aggregates of asphaltene-asphaltene units or single entities of asphaltene (monomers) that are environed, peptized, and consequently stabilized by resin components. This evaluation is based on quantum mechanical calculations through a DFT-D approach, and our emphasis is on refining energy parameters to establish a correlation between the number of asphaltene sheets and final stability of the system in a medium of asphaltene-resin components.

\section{Methodology}

The geometry of all structures was optimized through a DFT approach implemented in Gaussian 09. All geometry optimizations were performed at $298 \mathrm{~K}$ with default convergence criteria for residual forces and energy variations (displacements), without imposing any constraints or extra keyword options. To accurately describe $\pi$-stacking interactions, as well as to emphasize the importance of dispersion energy as a key factor in stabilizing the stacked dimers, dispersion-corrected DFT functional, B97-D ${ }^{[43]}$, associated with basis sets $6-31 \mathrm{G}^{*}$ and $6-311+\mathrm{G}^{* *}$, was selected as the level of DF calculations to optimize the structures. Recently, B97-D has been widely used to describe macro structures like asphaltene sheets and DNA-base pairs. $^{[44],[45],[46],[47],[48]}$ Counterpoise (CP) correction ${ }^{[49],[50]}$ was performed to limit basis set superposition error (BSSE) while studying intermolecular interactions. 
subtracting the total energy of the complex, $E_{\text {complex }}$, from the sum of its constituent parts, $E_{\text {fragment }}$, when they are in their lowest energy structure:

$E_{\text {bind }}=E_{\text {complex }}-\left(\sum E_{\text {fragment }}\right)$

Binding energy, indeed, reflects the net energy change through adsorption. However, in most cases the adsorption process is associated with the structural deformation of interacting fragments (mostly adsorbate).

The energy required for this deformation is almost supplied from the molecular binding of the adsorbate over the substrate as an energy gain process. In such situations, the actual value for stabilizing interactions is mostly compensated by structural deformation of the fragments. So, for the systems at which interacting constituents undergo major restructuring over interaction, the binding energy, $\mathrm{E}_{\mathrm{bind}}$, cannot be an appropriate descriptor for the characterization of interactions. On this basis, the interaction energy, $\mathrm{E}_{\text {int }}$, considering both binding and deformation energies, could be a reliable signifier for the strength of interactions in systems with substantial reconstruction. ${ }^{[51],[52],[53]}$ This term is calculated by the following equation:

$E_{\text {int }}=E_{\text {bind }}-\left(\sum \Delta E_{\text {fragment }}\right)$

where $\Delta E_{\text {fragment }}$ (sometimes shown with $\Delta E_{\text {deformation }}$ or $\Delta E_{\text {distorsion }}$ ) refers to the amount of energy required for the geometric distortion and electronic excitation of the fragments from their equilibrium ground state to the corresponding geometric and electronic structure in a complex.

\section{Models of asphaltene and resin monomers}

The morphology of asphaltene aggregates is a direct consequence of the asphaltene molecular structure and the particular orientation of asphaltene monomers through aggregation processing. Therefore, to present a clear picture of intermolecular interactions in asphaltene aggregation, our choice of asphaltene model is of prime importance. Although the structure of asphaltene is not unique, there are two commonly proposed molecular architectures for asphaltenes that are consistent with the results of experiments: archipelago, ${ }^{[54],[55],[56]}$ containing two or more separate polyaromatic hydrocarbons (PAHs) connected to each other through aliphatic side chains; and island, ${ }^{[57],[31]}$ containing a single unified PAH with 4-10 fused aromatic rings, with a centroid of the distribution around $7^{[58],[59]}$. This study used the island structure, based 
on the latest Yen-Mullins-modified model ${ }^{[60],[31]}$ proposed by Greenfield, ${ }^{[61]}$ including one pyrrolic nitrogen (a nitrogen atom embedded in a pyrrole ring, located in the aromatic part) ${ }^{[62]}$ as shown in Figure 1-a. Greenfield's modification was based on a rearrangement of some aliphatic chains of the Yen-Mullins model to reduce the pentane effect ${ }^{[63]}$ and its corresponding high internal energy. The pentane effect arises from bonding conflicts between atoms separated by six bonds that attempt to locate in a limited space for just six atoms, like a benzene or cyclohexane. The designation as the pentane effect comes from the five carbon and two hydrogen atoms involved in this conflict. Hydrogen atoms at the two ends of this seven-atom configuration overlap in a position suited for one atom, to form a cyclic conformer. A significant feature of asphaltene-pyrrole in this model is the non-planarity of the aromatic core, due to the pentane effect and the geometrical strain of neighboring rings. ${ }^{[61],[64],[65]}$

While asphaltenes consist of more than $48 \%$ aromatic carbons (in some cases even more than $~ 85 \%$ ) ${ }^{[66]}$, the content of aromatic carbon in petroleum resin is significantly lower ${ }^{[67]}$, which might be indicative of the less advanced aromatization in resins. In the current study, we used 6-methyl-dibenzothiophene as the resin molecule, based on the resin structure proposed by Murgich et al.[68] (Figure 1-b).

\section{Results and discussion}

In following sections, monomers of asphaltene and resin, and subsequently dimers of asphaltene are optimized and surveyed. The optimized structures are then used to build the starting structures in the next optimizations.

\subsection{Monomers of Asphaltene and Resin}

The first challenge for any type of theoretical study is the high molecular weight and large size of the asphaltene molecule, with the consequent computational cost. The first and most logical approach to this difficulty would be to simplify the structure. In the case of the asphaltene molecule, the extensive size of the aromatic region is responsible for the significant non-covalent interactions between asphaltene sheets and the formation of asphaltene aggregates. A reduced asphaltene without any aliphatic side chains retains its large size $\pi$-region and geometrical effect over self-association. Accordingly, to reduce the computational 
cost and enhance the number of samples (different arrangements), we deal with the reduced form of the asphaltene in some parts of the present calculations. However, side chains are still important, for the simple reason that petroleum asphaltene is composed of more than $48 \%$ aromatic carbons and the remaining is aliphatic carbons, as indicated by ${ }^{13} \mathrm{C}$ NMR studies ${ }^{[69]}$ So we compare the results arising from two structures, with and without aliphatic side chains, to give credence to the results obtained with the reduced structures.

Our calculation starts with refining the energetic and geometrical structure of the complete and reduced forms of an asphaltene-pyrrole monomer and a resin molecule. Optimization is carried out with B97-D functional and basis sets $6-31 \mathrm{G}^{*}$ and $6-311+\mathrm{G}^{* *}$, mentioned in the preceding section. Frequency calculation is carried out for each optimized molecule at the $\mathrm{HF} / 6-31 \mathrm{G}^{*}$ level to ensure being in a real minimum. The results of this optimization will be used in later sections.

Figure 1 also shows the charge distribution over the reduced form of asphaltene and a resin molecule, visualized through an electrostatic potential surface (ESP) map. The main priority of ESP visualization is to find the reactive sites of the molecules to design an effective interaction between two unit sheets of asphaltene or between asphaltene and resin. This map has been generated at the B97-D/6-31G* level, with isosurfaces of 0.01 electrons $a^{-3}$ through the Gauss View package. ${ }^{[70]}$ Sites with the lowest electrostatic potential energy value are in red, and sites with the highest are in blue, indicating the relative abundance and absence of electrons in these regions (negative and positive charge), respectively. This map vividly shows a positively charged region around peripheral hydrogens and a negatively charged region in the centers of reduced asphaltene, resin, pyrrolic nitrogen, and the sulfur of a thiophene group. We analyze this type of charge distribution in the formation of asphaltene-asphaltene and asphaltene-resin complexes in the following sections. 


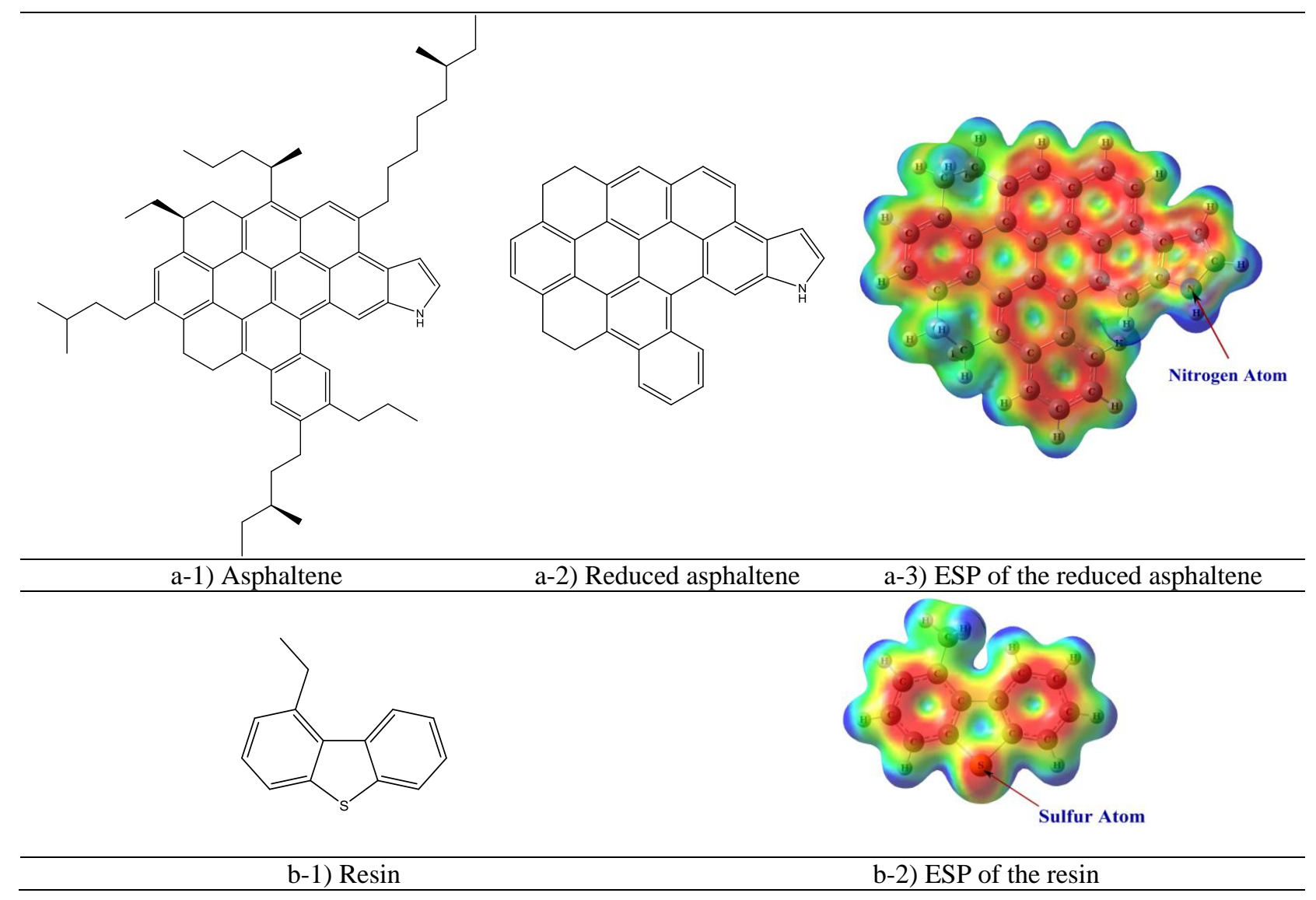

Figure 1. Optimized structures of (a-1) asphaltene-pyrrole molecule based on modified Yen-Mullins model, ${ }^{[61]}$ (a-2) reduced asphaltene-pyrrole molecule (excluding aliphatic chains), (b-1) 6-methyl-dibenzothiophene, the resin molecule proposed by Murgich et al ${ }^{[68]}$, and (a-3, b-2) electrostatic potential surface (ESP) for the reduced asphaltene and resin, respectively, generated at the $\mathrm{B} 97-\mathrm{D} / 6-31 \mathrm{G}^{*}$ level, scaled between -0.6 (red) to 37.7 (blue) $\mathrm{kcal} / \mathrm{mol}$. Red and blue colors signify the regions with charge accumulation and depletion, respectively.

\subsection{Asphaltene Dimers}

After preparation of an asphaltene monomer, modeling of an asphaltene dimer is the second step to simulate the process in which self-association of asphaltene molecules and their interactions with resin molecules occurs. To do so, the diversity of the probable structures is one of the limitations. This diversity arises from the different arrangements of the asphaltene sheets with respect to each other. The presence of heteroatoms in asphaltene sheets and also in peripheral chains causes a wider intermolecular orientation

242 distribution even for the simulation of a dimer, the simplest form of asphaltene aggregate. There are four most commonly proposed arrangements for an asphaltene dimer: parallel or face-to-face $(\pi-\pi$ interaction); 
parallel-displaced or offset ( $\sigma-\sigma$ interactions); T-shape or face-to-edge ( $\pi$ - $\sigma$ interactions); and T-shape or edge-to-edge ( $\sigma-\sigma$ interactions). ${ }^{[71],[72],[73]}$

Among the various conformations examined here, Table 1 shows optimized structures concerning three preferential orientations: parallel, offset, and diagonal. Because of a negligible difference between corresponding structures at two basis set levels $\left(6-31 G^{*}\right.$ and $\left.6-311+G^{* *}\right)$, just one series of structures is shown in this table. The energy information and the optimum distance between two asphaltene sheets have also been reported in this table. Binding distances reported in this table are the vertical distance $\left(\mathrm{d}_{\text {ver }}\right)$ between two approximate centers of asphaltene sheets, and the optimum distance between two mass centers of asphaltene sheets (d).

Table 1. Three lowest energy structures of asphaltene-pyrrole dimer, and corresponding relative energy $\left(\Delta \mathrm{E}_{\mathrm{tot}}\right)$ of different conformations with respect to the lowest energy structure, binding energy $\left(\mathrm{E}_{\text {bind }}\right.$ ), binding distance between two asphaltene mass centers (d), and vertical binding distance between two approximate centers of asphaltene sheets $\left(\mathrm{d}_{\mathrm{ver}}\right)$. Energies are counterpoise corrected and reported in $\mathrm{kcal} / \mathrm{mol}$.

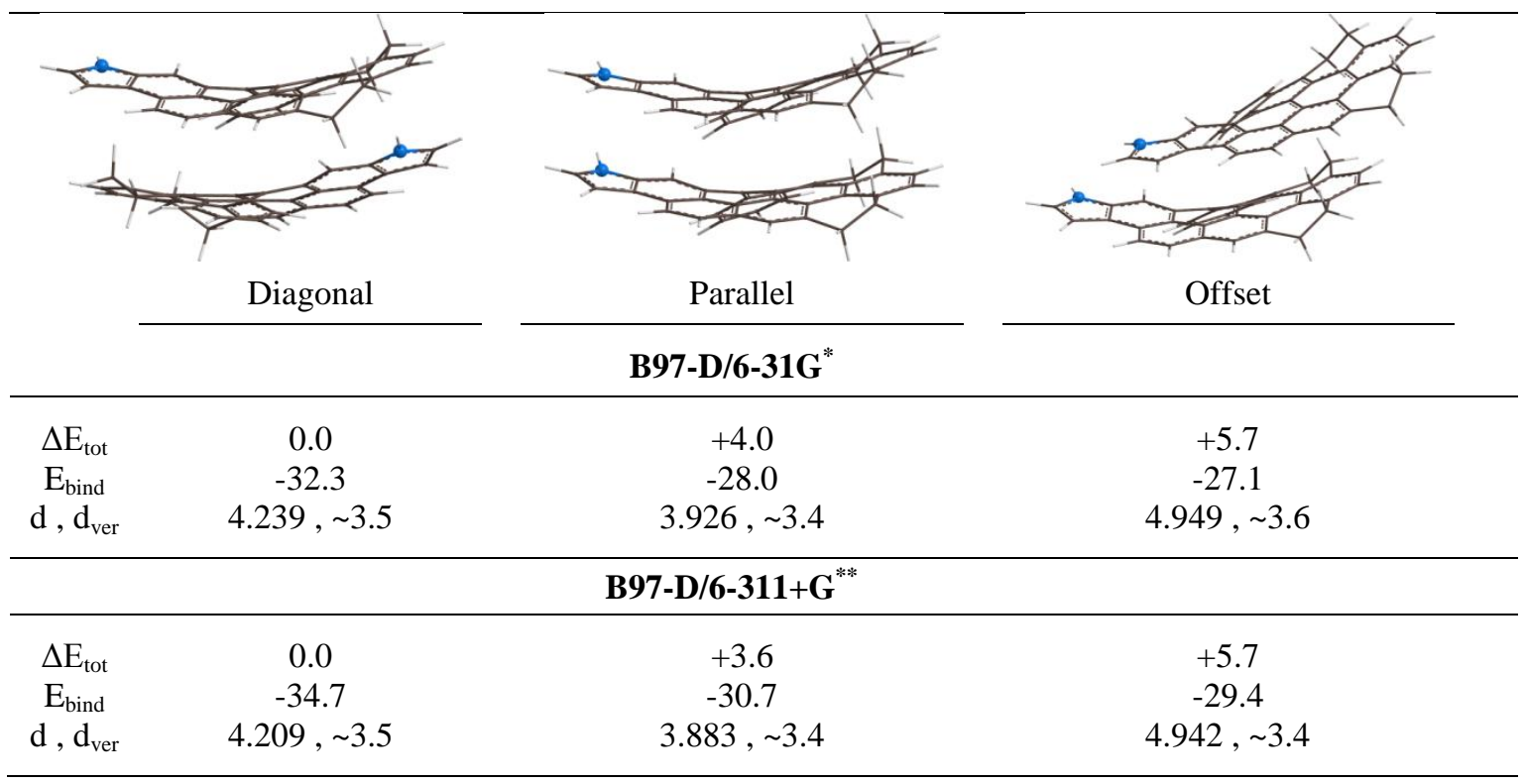

In Table 1, relative energy $\left(\Delta \mathrm{E}_{\mathrm{tot}}\right)$ is defined with respect to the total energy of the lowest energy structure (diagonal), and binding energy $\left(\mathrm{E}_{\text {bind }}\right)$ is related to the thermodynamic stability of the system. Due to the stiffness and rigidity of the polyaromatic rings of individual asphaltene molecules, they do not notably deform over dimerization, and their corresponding deformation energy is small. As an example, the 
deformation energy of a diagonal structure is $1.4 \mathrm{kcal} / \mathrm{mol}$ (not tabled). Therefore, based on Equation 2, the binding energy and interaction energy are somewhat the same, and binding energy could be also considered as a representative of the strength of interaction between two asphaltene sheets. Based on the values reported in Table 1, the range of energy is from -29.4 to $-34.7 \mathrm{kcal} / \mathrm{mol}$ at the $\mathrm{B} 97-\mathrm{D} / 6-311+\mathrm{G}^{* *}$ level, and with a very small difference (about $2 \mathrm{kcal} / \mathrm{mol}$ ) from -27.1 to $-32.3 \mathrm{kcal} / \mathrm{mol}$ at the B97-D/6-31G ${ }^{*}$ level.

The energy values reported here seem to be remarkably lower than $-55 \mathrm{kcal} / \mathrm{mol}$, the value reported by Pacheco-Sánchez et al.[73] for one asphaltene dimer in a vacuum using COMPASS Force Field, [74], [75] derived using classical Molecular Mechanics (MM) for a parallel arrangement with binding distance of 4 and $5 \AA$. In another study ${ }^{[76]}$ based on $\mathrm{MM}$ and semiempirical calculations, the interaction energy was reported to be $-45 \mathrm{kcal} / \mathrm{mol}$ for two asphaltene dimers, each containing a hydroxyl group. A small percentage of the difference between DFT results and MM results could be attributed to the removal of aliphatic side chains in our calculations, and a portion of the difference may be caused by the inadequacy of the level of calculations based on COMPASS Force Field parameters.

To examine this possibility, we added the side branches of real asphaltene to the reduced asphaltene dimer in its most stable conformer (the diagonal dimer), and compared the results, as shown in Table 2. Due to the high computational cost of the Gaussian package to (structurally) optimize the branched asphaltene dimer, we resorted to other software with higher computational speed. Geometric optimization of the branched asphaltene was carried out by a DFT-based $\mathrm{DMol}^{3}$ module ${ }^{[77],[78]}$ embedded in Accelrys Materials Studio using Perdew-Burke-Ernzerhof $(\mathrm{PBE})$ functional ${ }^{[79]}$ with Grimme's dispersion correction scheme ${ }^{[44]}$ and a double numerical plus polarization (DNP) basis set (comparable to a Gaussian 6-31G ${ }^{* *}$ basis set). $\mathrm{DMol}^{3}$ benefits from numerical basis functions that reduce the practical computational time compared to Gaussian-based methods. The energy of this optimized structure was calculated via B97-D/6-31G ${ }^{*}$ in the Gaussian package. Binding energy values (-36.7 vs. $-32.3 \mathrm{kcal} / \mathrm{mol})$ for two asphaltene dimers, with and without aliphatic side chains, reinforce the aforementioned explanation that MM-based results are associated with some overestimation on the binding energy value. 
Table 2. Comparing asphaltene dimers in the presence and absence of aliphatic side chains. Binding energies $\left(\mathrm{E}_{\mathrm{bind}}\right)$ are counterpoise corrected and reported in $\mathrm{kcal} / \mathrm{mol}$. Binding distances between two asphaltene centers (d) and vertical distances $\left(\mathrm{d}_{\mathrm{ver}}\right)$ are in $\AA$.

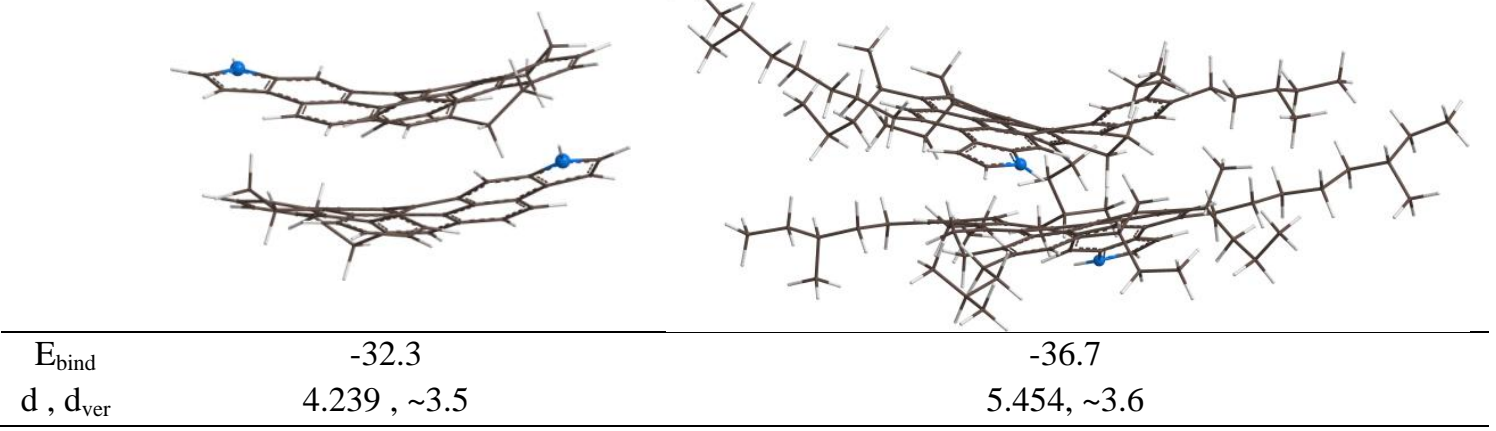

On the other hand, the bond distance calculated between the centers of mass of asphaltene sheets was found to be around $4-5 \AA$, and the vertical distance to be $3.5 \AA$, which are in good agreement with X-ray diffraction and small angle scattering results reported by Yen et al., ${ }^{[2],[80]}$ who reported the interlayer distance between two asphaltene sheets to be 3.55-3.70 $\AA$. In addition, our result is comparable with those from Molecular Dynamics (MD) simulations that reported an interlayer distance of 3.5-4 $\AA^{[73], ~[81]}$

The stability of the systems is another notable point in Table 1 . As shown in this table, the most stable structure is the diagonal structure, in which two nitrogen atoms are at the maximum distance from each other (located in opposite corners of a rhombus frame), with energy of -34.7 kcal/mol at the B97-D/6$311+\mathrm{G}^{* *}$ level, although the binding distance between two monomers is not the lowest of the three proposed structures. Comparison of the other two structures (parallel and offset) shows that these two structures are not very different energetically; with a small energy difference, the parallel dimer is more stable than the offset dimer. At the B97-D/6-311+G** level, the energy difference between the two structures is not more than $1.3 \mathrm{kcal} / \mathrm{mol}$.

Based on the ESP map in Figure 1, the negative charge (red color) accumulation in the center of the asphaltene molecule increases the contribution of electrostatic repulsion between two asphaltene sheets, due to the $\pi$-electron cloud, which, in turn, should reduce the stability of parallel structures, while our results confirm that parallel conformers are the most stable structures (note that the diagonal structure is a parallel structure in nature). Indeed, stacking interaction is favorably affected by a nitrogen heteroatom attached to 
the aromatic core of asphaltene. The positive role of a nitrogen atom in stabilizing some particular asphaltene dimers is attributed to the Hunter-Sanders ${ }^{[71],[82]}$ rule and the electron-withdrawing nature of this atom, which can pull the negative charge of the ring $\pi$-electrons and provide a favorable $\pi$ stacking interaction. Thus, the polarization of pi-electron density due to the presence of electron-withdrawing groups on aromatic rings as well as the induced electrostatic forces (London dispersion) are attraction forces that favor stacking the asphaltene planes.

In addition, the greater stability of a diagonal arrangement, which is a typical parallel structure with the maximum distance between two nitrogen atoms, compared to a parallel structure at which nitrogen atoms are very close and in front of each other, make us believe that at greater distances, the electrostatic interaction of the two nitrogen atoms with distinct partial charges is reduced, and the positive role of nitrogen becomes pronounced.

\section{T-shaped conformers}

Another notable point in Table 1 is the absence of the T-shape as one of the expected structures for an asphaltene dimer. A tilted T-shape with the minimum electron repulsion between two fragments is the most favorable conformer for simple $\pi$-stacking structures like a benzene dimer, ${ }^{[83],[84],[85],[45]}$ whereas theoretical studies on PAHs show that parallel or nearly parallel stacking structures are preferred over Toriented structures. ${ }^{[86], ~[28]}$ This could be attributed to the steric repulsion between aliphatic side chains of two molecules in T-shape structures. ${ }^{[87]}$

In the case of our study, at the B97-D level, irrespective of the temperature range applied (not tabled) and even in the absence of aliphatic chains, no T-conformation was observed for asphaltene, and all different initial T-shape structures rearranged to the offset $\pi$-stacked geometries. To be confident of accurate identification of the geometric preference, all starting T-shaped structures were reexamined under the M06$2 \mathrm{X}$ functional, which also benefited from the improved dispersion correction. Except for one case (a lower energy structure with intermolecular orientation angle $\sim 30^{\circ}$ ), almost all starting T-conformers rearranged into parallel-displaced, indicative of the priority of $\pi-\pi$ stacking over $\pi-\sigma$ interactions in the perpendicular position. 
343

344

345

346

347

348

349

350

351

352

353

354

355

356 hypothetical micelle.

\subsection{Micellar structure of asphaltene: pros and cons}

Referring to a "micelle" ("a surfactant micelle" in its traditional definition) as an asphaltene nanoaggregate in crude oil, bitumen, or organic solvents has encountered opposition in the last few years. ${ }^{[29],[31],[30]}$ However, the colloidal hypothesis of asphaltenes is still one of the most generally accepted and commonly used viewpoints about the state of asphaltene in crude oil (or in bitumen). ${ }^{[12],[13],[14],[15],[16],[17]}$

In the colloidal model, components with the greatest molecular weight and highest aromaticity (like asphaltene) are located in the center of micelles, and lighter components with less aromatic nature (such as aromatics and saturates) constitute outer shells. Resin molecules are supposed to be onto the surfaces or into the interiors (gap spaces) of asphaltene molecules. ${ }^{[13],[19],[88]}$ In the surfactant micellar model, the highly dispersive character of resin-asphaltene micelles is attributed to the surfactant-like property of resins, which contain polar functional groups such as carbonyl, aldehyde, amine, etc. On this basis, the stability of asphaltene aggregates in hydrocarbon solutions is guaranteed by resin molecules. Figure 2 shows the arrangement of SARA (the acronym of saturates, aromatics, resins, and asphaltene) fractions in one
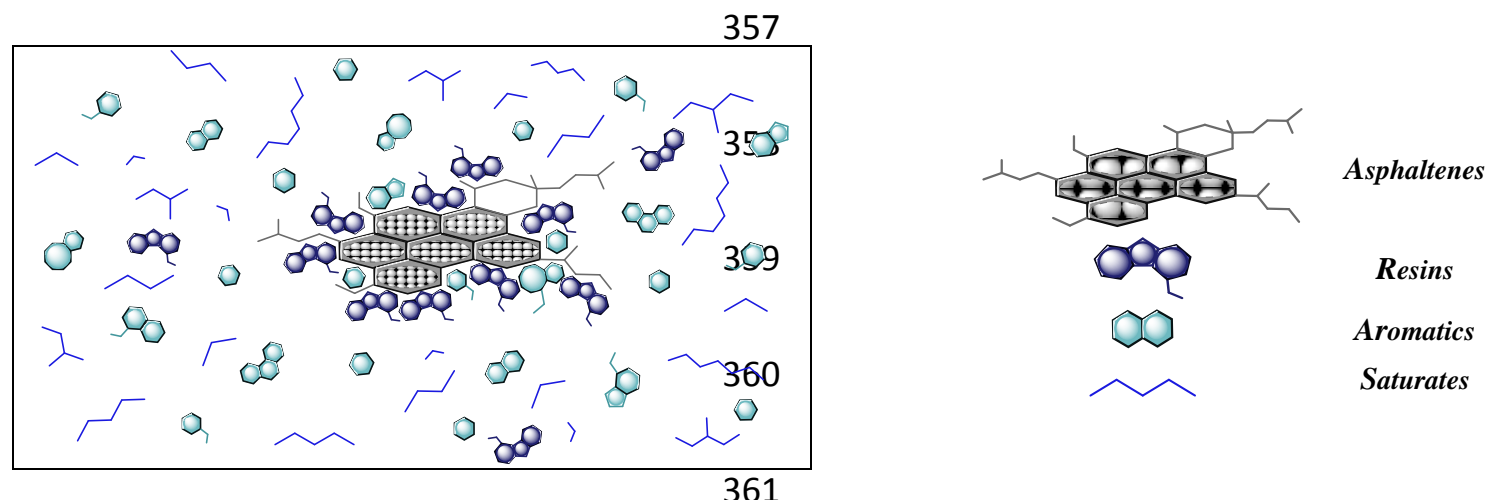

Figure 2. Colloidal suspension of one hypothetical micelle of asphaltene in a dispersing medium of lighter resins, aromatics and saturates

Although opponents of the surfactant model believe that resins are not surfactants and that colloidal suspensions of asphaltene are still formed in solvent models even in the absence of resin molecules, they do not reject some degree of interaction between asphaltene and resin. ${ }^{[31]}$ Accordingly, steric repulsion between 
asphaltene molecules is a factor that increases the maintenance of the asphaltene particles at a distance sufficient to inhibit coalescence. In the opposition group, Mullins ${ }^{[31]}$ has a more positive look at the issue. He accepts the colloidal view of asphaltene aggregates and that there is some degree of interaction between asphaltene and resin, but he (along with some other researchers ${ }^{[29],[89],[30]}$ ) rejects the surfactant-like or peptizing role of resin molecules in the stability of asphaltene colloidal suspensions.

There seems to be a general consensus on the colloidal suspension of asphaltenes, even if they are different from the "micelle" in its traditional definition. As stated in the introduction section, in this study, the term "micelle" is used for a molecular aggregate depicted in Figure 2, which is a cluster composed of an asphaltenic core surrounded by other fractions like lighter resins, aromatics, and saturates, irrespective of the role of resin molecules and whether they act as surfactants.

A question often posed about the nature and composition of each individual micelle is whether asphaltenes exist as stacked layers within the micelle or as single molecular units surrounded by resin molecules. Simply stated, are there suspended particles of asphaltene-asphaltene aggregates surrounded by resins, or are there single layers of asphaltene (i.e., non-stacked layers) peptized and consequently stabilized by resins? In the third part of this study (which is the main purpose of the present work), we try to find a solution for the posed question and to investigate the probable structure of the asphaltenic core, to answer whether one or more than one asphaltene exist per micelle in vacuum residue and asphalt. This evaluation is based on refining the energy parameters to establish a correlation between the number of asphaltene sheets and the final stability of the system in a medium of asphaltene and resin components. To do so, we set up interacting models for all required pairs of asphaltene and resins: asphaltene-resin (A-R) and asphalteneasphaltene-resin (A-A-R).

\subsubsection{Interaction of resin and asphaltene}

The structural similarity between a resin molecule and asphaltene (resins are smaller, with lower molecular weight) leads us to investigate $\pi-\pi$ stacked (face-to-face) conformations as the most efficient interacting geometries between the two components asphaltene and resin, as we found for asphalteneasphaltene. In another DFT study, Alvarez-Ramirez et al. ${ }^{[90]}$ reported face-to-face orientation as the preferred 
configuration for resin and asphaltene molecules. In the current study, the proposed structure, 6-methyldibenzothiophene, by Murgich et al., ${ }^{[68]}$ was considered and optimized as the resin molecular structure.

Based on molecular mechanical calculations, ${ }^{[28]}$ the formation of molecular aggregates of asphaltene and resin in a colloidal medium is due to the suitable fitting between aromatic planes of two fragments (as the action of a lock and key) and the lowest steric repulsion between the peripheral chains. We took into account this point while designing the initial structures of asphaltene-resin complexes to be optimized. The most stable structures of each component (resin and asphaltene) were used to build the starting structures of resin-asphaltene complexes. Full geometric optimization, without imposing any constraints, was performed to find the lowest energy structures. Calculations were performed at the B97-D/6-31G* level.

To construct our interacting model of asphaltene-resin, the number of resin molecules required to interact with an asphaltene target is of particular importance. Resin molecules are smaller and lower in molecular weight compared to asphaltene molecules, so the asphaltene micelles are expected to be

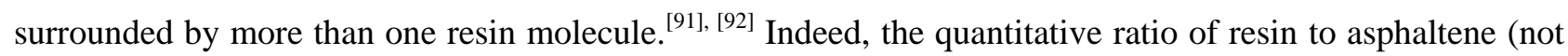
all the resin molecules participate in the formation of micelles) characterizes the colloidal stability of VR, or the onset of destabilization of the system and precipitation or phase separation. In addition to asphaltene(s) and a fraction of resin molecules, the intra-micelle space is occupied by other fractions like aromatics and saturates. The size of the asphaltene core and the number of aromatic fused rings, which are indicative of the active sites, determine the number of resin molecules surrounding the asphaltenic core. With these considerations, three or four resin molecules may fulfill the requirements of our model, as shown in Table 3 .

Table 3 displays energy information for the interaction between a single asphaltene (branched and reduced forms) and resin molecules. As shown, increasing the number of resin molecules increases the thermodynamic stability of the system; when the number of resins is increased from one to three, binding energy is increased about three times (from -17.0 to $-47.2 \mathrm{kcal} / \mathrm{mol}$ ). This correlation between thermodynamic stability of the complex and the number of resin molecules is strongly supported by the experimental evidence: the greater the number of resins, the higher the colloidal stability. As stated above, the colloidal stability of the vacuum residue relies on the ratio of resin to asphaltene. 
Table 3. Interaction between a single asphaltene (reduced and branched forms) and resin molecules. Binding energies

423 are counterpoise corrected and reported in $\mathrm{kcal} / \mathrm{mol}$. Asphaltene and resin are denoted by $\mathbf{A}$ and $\mathbf{R}$, respectively.

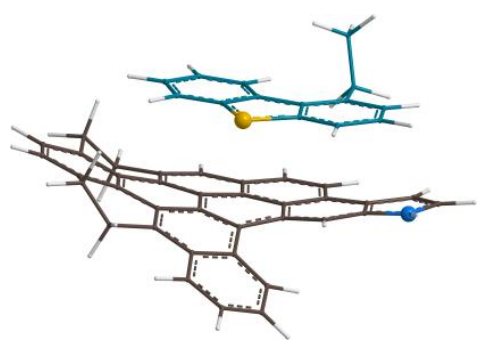

A-R(monomer)

$\mathrm{E}_{\text {bind }}=-17.0$

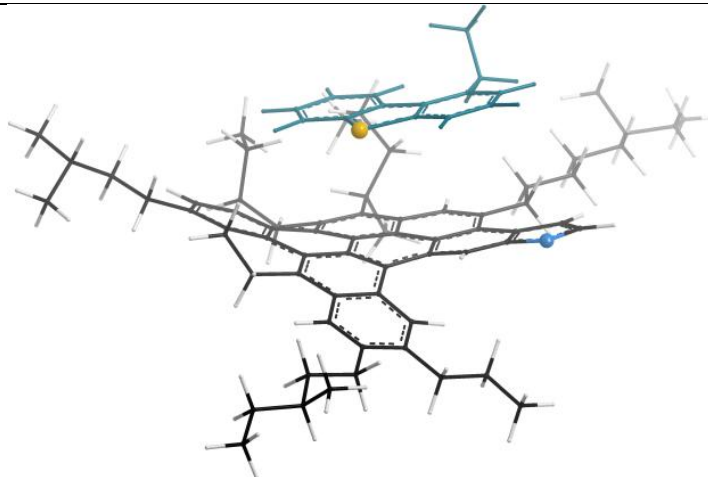

A-R(monomer)

$\mathrm{E}_{\text {bind }}=-19.4$

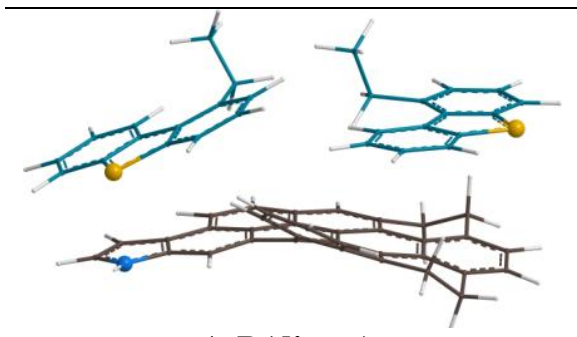

A-R(dimer)

$\mathrm{E}_{\text {bind }}=-27.5$

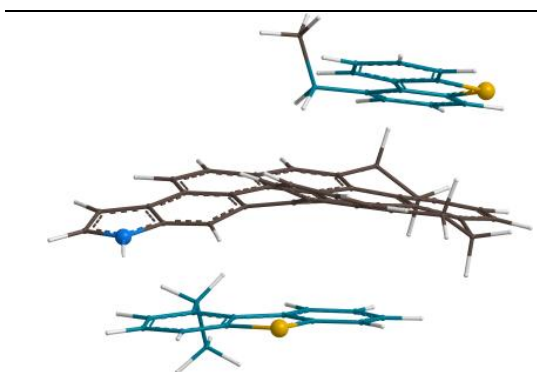

A-R(dimer)

$\mathrm{E}_{\text {bind }}=-32.4$

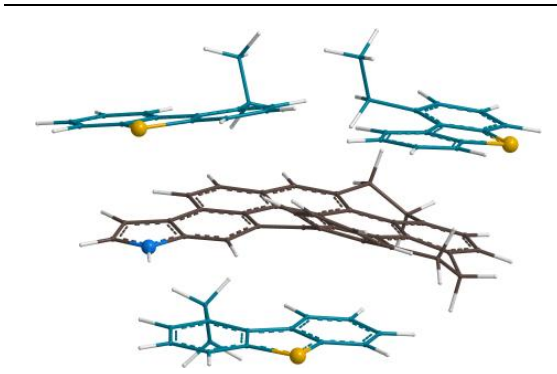

A-R(trimer)

$\mathrm{E}_{\text {bind }}=-47.2$

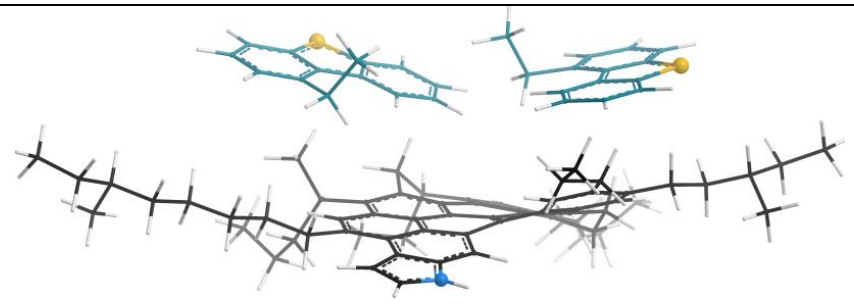

A-R(dimer)

$\mathrm{E}_{\text {bind }}=-35.5$

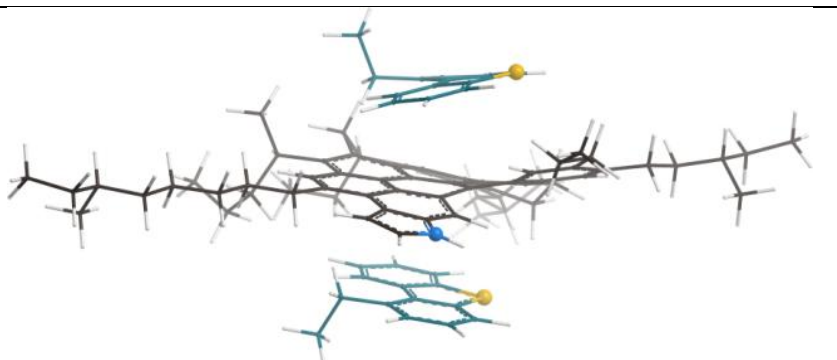

A-R(dimer)

$\mathrm{E}_{\text {bind }}=-38.12$

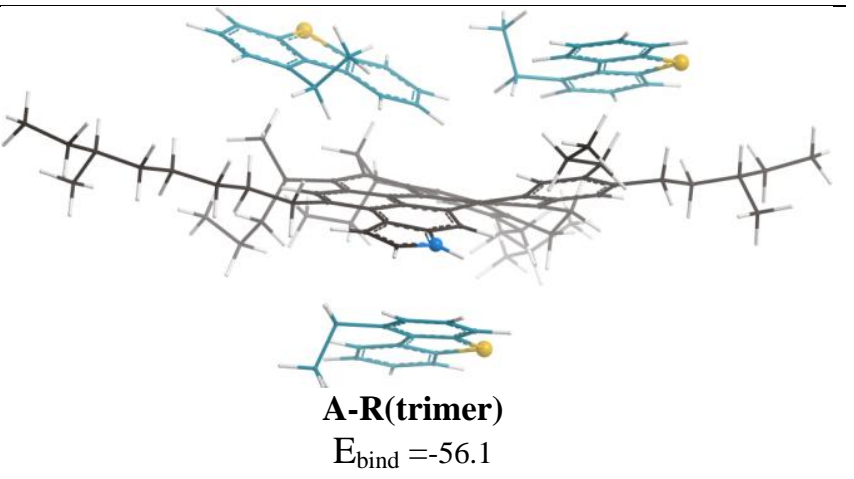

Comparison between the energy values of an asphaltene dimer in Table 1 and the reduced structures 
interactions are preferred over asphaltene-asphaltene, only if the number of resin molecules is greater than the number of asphaltene molecules. Accordingly, while the binding energy for an asphaltene dimer (in the most stable conformer) is $32.3 \mathrm{kcal} / \mathrm{mol}$, the binding energy of an asphaltene-resin trimer, A-R(trimer), reaches $47.2 \mathrm{kcal} / \mathrm{mol}$.

Our results in this part with asphaltene - asphaltene micelles and asphaltene - resin micelles also provide a reasonable answer about the homogeneous or heterogeneous nature of the micelles we encounter. ${ }^{[14],[33],[93]}$ Stronger interactions in asphaltene-resin complexes (in high ratios of resin to asphaltene) reinforces the postulation that micelles are heterogeneous in nature if the number of resin molecules is greater than the number of asphaltene molecules.

Reduced structures of asphaltene-pyrrole might be blamed for the absence of aliphatic chains and exaggerated behaviors of stacking, which could affect its willingness towards other molecules like resins, and question the validity of our results. To add credence to the use of reduced structures of asphaltenepyrrole, and to verify that these structures can simulate the behavior of branched structures, we also calculated interaction energies for asphaltene-resin monomers in the presence and absence of peripheral groups of asphaltene. The results are also shown in Table 3. A simple comparison indicates that the reduced structures of asphaltene generate more than $84 \%$ of the total interaction energy arising from branched structures. The atoms responsible for this contribution are aromatic carbons located in the center of an asphaltene monomer, highlighting the importance of the ring-ring interactions between resin and asphaltene.

Nevertheless, the role of aliphatic side chains in stabilizing the complex is undeniable, in particular for large systems. As an example, in an asphaltene-resin (trimer), A-R(trimer), the contribution of the side chains to stability of the system is $8.9 \mathrm{kcal} / \mathrm{mol}$. This is in line with Takanohashi et al.'s results, which emphasize the stabilizing effects of aliphatic side chains. ${ }^{[94]}$

Furthermore, we should point out the two different arrangements proposed for an asphaltene-resin (dimer), A-R (dimer). As shown in Table 3, the structure in which two resin molecules are arranged in symmetrical positions (above and below asphaltene) is energetically more favorable than the scenario in which both resins are on the same side of the asphaltene surface: -32.4 versus $-27.5 \mathrm{kcal} / \mathrm{mol}$ for the reduced forms, and -38.12 versus -35.5 for the branched structure. This can be attributed to the extent of $\pi-\pi$ overlap 
between the fused aromatic rings of asphaltene and the aromatic region of resin molecules; this overlap is significantly higher when two resin molecules are arranged on opposite sides of the asphaltene molecule compared to having both resins on the same side. This observation further indicates the importance of ringring interactions between resin and asphaltene molecules.

To pursue our primary objective in this study, we increased the dimension of the asphaltenic core to two asphaltene molecules per micelle and investigated the influence of resin molecules on the stability of the new structures. The results are shown in Table 4. In this table, all structures have been optimized at the B97$\mathrm{D} / 6-31 \mathrm{G}^{*}$ level. Based on the asphaltene-resin (dimer) results in Table 3, only the arrangement that led to the most stable structure (two resins located on opposite sides of the asphaltene surface), was examined and is reported in Table 4.

Table 4. Interaction between an asphaltene dimer and resin molecules. Binding energies are counterpoise corrected and in $\mathrm{kcal} / \mathrm{mol}$. Asphaltene and resin are denoted by $\mathbf{A}$ and $\mathbf{R}$, respectively.

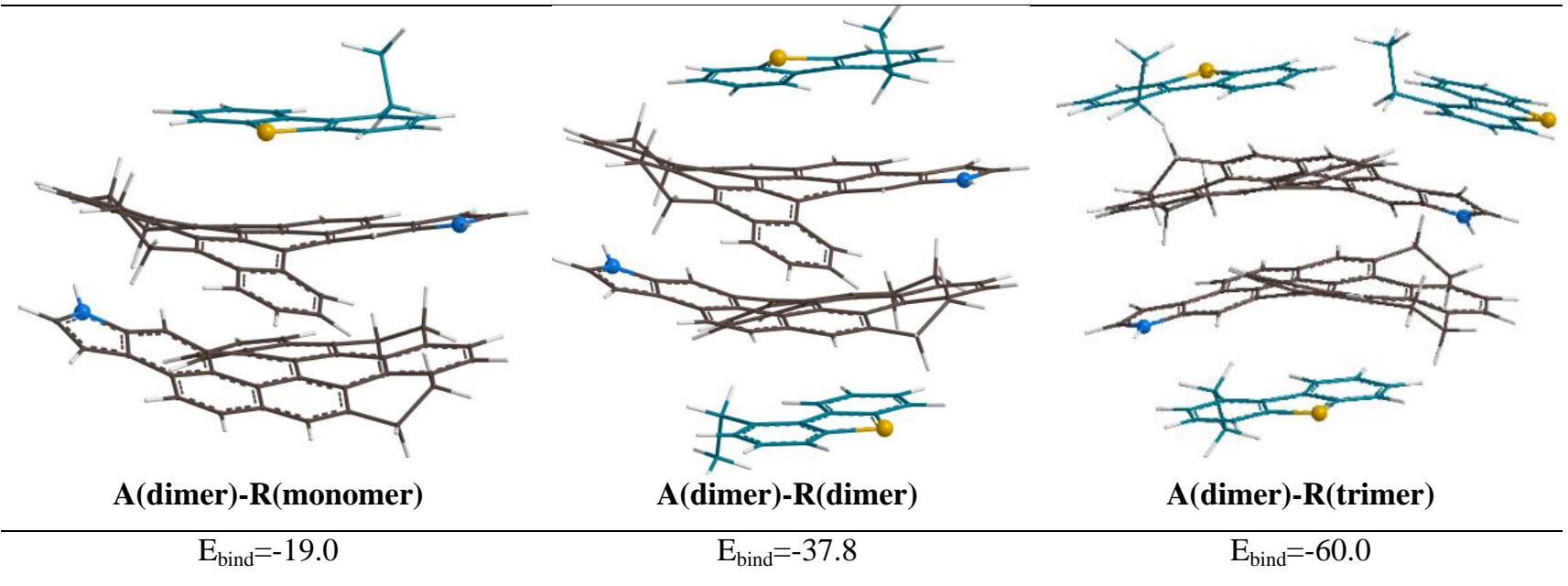

We are now in a position to take a look at the results in Table 3 (as a micellar model with monolayer asphaltenic core) and Table 4 (as a micellar model with double layer asphaltenic core), which provide valuable points of comparison. Energy values reported in the tables clearly show that resin molecules stabilize asphaltene dimers better than asphaltene monomers.

For example, in structures enriched with resin (including three resin molecules), binding energy increases from -47.2 to $-60.0 \mathrm{kcal} / \mathrm{mol}$ (a $27 \%$ increase), indicative of more stability of the structure with the 
double-layer asphaltene compared to that of the single-layer asphaltene. The same trend is observed for other structures. Our results give more credence to the idea that micellar behavior of crude oil is better described by stacked layers of asphaltene-asphaltene environed by resin molecules, rather than non-stacked single layers of asphaltene.

As the last part of our study, we decided to investigate the interaction between two asphaltene unit sheets in the presence and absence of resin molecules. To do so, different fragmentation has been carried out. To get an easier understanding of the interactions, we have shown the interacting components as separate elliptical planes in Table 5.

Table 5. Interaction between two asphaltene sheets in the absence and presence of resin molecules;. To get more convenient understanding, interacting components are shown by separate elliptical planes. $d$ is the distance between two asphaltene centers. Counterpoise corrected energies are binding energy $\left(\mathrm{E}_{\mathrm{bind}}\right)$, deformation energy of fragments $\left(E_{\text {def }}\right)$, and interaction energy between two asphaltene planes $\left(E_{\text {int }}\right)$. Energies and distances are in $\mathrm{kcal} / \mathrm{mol}$ and $\AA$, respectively.
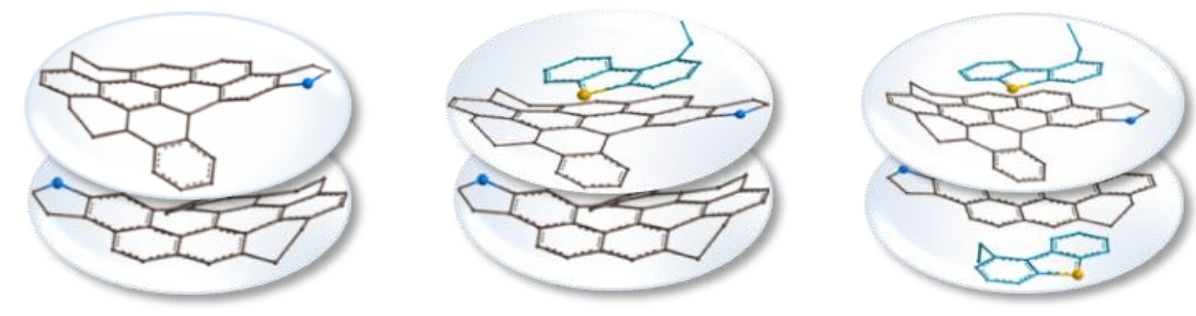

$\mathbf{A}+\mathbf{A}$

$\mathbf{A}+\mathbf{A}$
$\mathrm{d}=4.239$

$\mathbf{R}-\mathbf{A}+\mathbf{A}$ $\mathrm{d}=3.746$

$\mathrm{E}_{\text {bind }}=-32.3$ $E_{\text {bind }}=-37.6$

$\mathrm{E}_{\mathrm{def}}=+1.44$ $\mathrm{E}_{\mathrm{def}}=+3.2$

$\mathrm{E}_{\mathrm{int}}=-33.7$
$\mathbf{R}-\mathbf{A}+\mathbf{A}-\mathbf{R}$ $d=3.737$ $E_{\text {bind }}=-34.5$ $E_{\text {def }}=+3.4$ $\mathrm{E}_{\mathrm{int}}=-37.9$

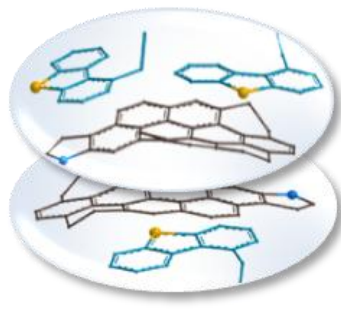

R-R-A + A-R $d=3.709$ $E_{\text {bind }}=-34.4$ $\mathrm{E}_{\mathrm{def}}=+5.1$ $\mathrm{E}_{\mathrm{int}}=-39.5$

In Table 5, the first notable point is the significant reduction of binding distance (d) between two asphaltene centers from adding resin molecules: addition of the first resin to an asphaltene dimer reduces the binding distance from 4.239 to $3.746 \AA$. This reduction of distance is associated with an increase in interaction energy from -33.7 to $-40.8 \mathrm{kcal} / \mathrm{mol}$. The presence of the resin component and the positive 
evolution of the system towards the enhancement of interaction between the planes corroborate the experimental findings that the dispersive character of asphaltene micelles is increased in the presence of resin. In contrast to binding distance, no regular trend is observed in correlation between the number of resin molecules and reported energies. This might be due to the fact that except for the bare asphaltene dimer, which is in its lowest energy state (examined in section 3.2), the others may not be in their lowest energy structures, though they have been completely optimized.

\section{Conclusion}

The interplay between asphaltene and resin molecules in the formation of micelles and consequently the identification of the micellar structure of asphaltenes have been a matter of some speculation and intense debate in recent years. One of the issues discussed is that formation of coherently small asphaltene micelles in high ratios of resin/asphaltene might indicate that these suspended particles are individual entities of asphaltene (monomers) that are environed by resin components, and not necessarily stacked aggregates of asphaltene-asphaltene units.

Given a substantial body of experimental and somewhat MD reports on the physics of asphaltene micelles, we examined this subject from a different perspective using rigorous quantum mechanical calculations. As the building blocks of our study, we used one of the Yen-Mullins-modified structures ${ }^{[60],[31]}$ proposed by Greenfield ${ }^{[61]}$ as the asphaltene molecule, and 6-methyl-dibenzothiophene, proposed by Murgich et al., ${ }^{[68]}$ as the resin molecule. While our initial comparison was conducted on a reduced form of asphlatene (without aliphatic side chains), we further examined the full structures (including side chains) to determine the relative effect of aliphatic side chains on colloidal stability. Based on our quantitative findings, the reduced forms of asphaltene reproduce more than $84 \%$ of the total interaction energy arising from the branched structures. This further indicates the importance of the Van der Waals and $\pi-\pi$ interactions associated with the aromatic core of the two components.

To find a correlation between the number of asphaltene sheets and the final stability of the system after their interaction with resin molecules, we set up interacting models for all required pairs of asphaltene and resins: asphaltene-resin (A-R) and asphaltene-asphaltene-resin (A-A-R). The calculated energy values 
clearly show that resin molecules stabilize asphaltene dimers better than asphaltene monomers. Simply stated, the micellar behavior of crude oil is better described by asphaltene-asphaltene associations.

In support of the role of resins, the binding distance between the center of mass of two asphaltene sheets is notably reduced in the presence of resin molecules. The addition of the first resin molecule to an asphaltene dimer reduces the binding distance from 4.239 to $3.746 \AA$; this value is further reduced to 3.709 $\AA$ when the number of resins in the complex is increased to three. This adds more credence to the postulation that resin molecules increase the effective interaction between asphaltene sheets.

Moreover, our calculations show that if both asphaltene and resin are present, resin-asphaltene interactions are preferred over asphaltene-asphaltene interactions, only if the number of resin molecules is more than the number of asphaltene molecules. This is in line with experimental evidence indicating that the colloidal stability of petroleum residua relies on the ratio of resin to asphaltene: thermodynamic stability of VR is achieved only when the relative concentration of resin is higher than that of asphaltenes.

\section{ACKNOWLEDGMENT}

The authors would like to acknowledge the support provided by the National Science Foundation, Award No: 1546921 and 1150695). The contents of this paper reflect the view of the authors, who are responsible for the facts and the accuracy of the data presented. 
[1] Mitchell DL, Speight JG. The solubility of asphaltenes in hydrocarbon solvents. Fuel 1973;52:149-52.

554

[2] Yen TF, Erdman JG, Pollack SS. Investigation of the structure of petroleum asphaltenes by X-ray diffraction. Anal Chem 1961;33:1587-94.

[3] Oberlin A. High-resolution TEM studies of carbonization and graphitization, In Chemistry and physics of carbon, vol. 22; Thrower PA editor, 1989, 2-135.

[4] Oberlin A, Bonnamy S, Rouxhet P. Colloidal and supramolecular aspects of carbon, In Chemistry and Physics of Carbon, vol. 26; Thrower PA and Radovic LR editors. Marcel Dekker, New York; 1999, 2-139.

[5] Groenzin H, Mullins OC. Asphaltene molecular size and structure. J Phys Chem A 1999;103:11237-45.

[6] Groenzin H, Mullins OC. Molecular size and structure of asphaltenes from various sources. Energy Fuels 2000;14:677-84.

[7] Sharma A, Mullins OC. Insights into molecular and aggregate structures of asphaltenes using HRTEM. In Asphaltenes, Heavy Oils, and Petroleomics, Mullins OC, Sheu EY, Hammami A, and Marshall AG editors: Springer; 2007. p. 205-29.

[8] Indo K, Ratulowski J, Dindoruk B, Gao J, Zuo J, Mullins OC. Asphaltene nanoaggregates measured in a live crude oil by centrifugation. Energy Fuels 2009;23:4460-9.

[9] Sirota EB. Physical structure of asphaltenes. Energy Fuels 2005;19:1290-6.

[10] Witherspoon PA. Studies on petroleum with the ultracentrifuge. Report of investigations no 2061958.

[11] Redelius PG. The structure of asphaltenes in bitumen. Road Mater Pavement 2006;7:143-62.

[12] Murgich J. Molecular simulation and the aggregation of the heavy fractions in crude oils. Mol Simul 2003;29:451-61.

[13] Li S, Liu C, Que G, Liang W. Colloidal structures of vacuum residua and their thermal stability in terms of saturate, aromatic, resin and asphaltene composition. J Petrol Sci Eng 1999;22:37-45.

[14] Andersen SI, Speight JG. Petroleum resins: separation, character, and role in petroleum. Petrol Sci Technol 2001;19:1-34.

[15] Spiecker PM, Gawrys KL, Trail CB, Kilpatrick PK. Effects of petroleum resins on asphaltene aggregation and water-in-oil emulsion formation. Colloid Surface A 2003;220:9-27.

[16] Li S, Liu C, Que G, Liang W, Zhu Y. A study of the interactions responsible for colloidal structures in petroleum residua. Fuel 1997;76:1459-63.

[17] Lesueur D. The colloidal structure of bitumen: Consequences on the rheology and on the mechanisms of bitumen modification. Adv Colloid Interface Sci 2009;145:42-82.

[18] Nellensteyn FJ. The constitution of asphalt. J Inst Pet 1924;10:311-25.

[19] Pfeiffer JP, Saal RNJ. Asphaltic bitumen as colloid system. J Phys Chem 1940;44:139-49.

[20] Saal RNJ, Labout JWA. Rheological Properties of Asphaltic Bitumen. J Phys Chem 1940;44:149-65. 
[21] Galtsev VE, Ametov IM, Grinberg OY. Asphaltene association in crude oil as studied by ENDOR. Fuel 1995;74:670-3.

[22] Escobedo J, Mansoori GA. Viscometric principles of onsets of colloidal asphaltene flocculation in paraffinic oils and asphaltene micellization in aromatics. SPE Prod Facil 1997;12:116-22.

[23] Mullins OC. The asphaltenes. Annu Rev Anal Chem 2011;4:393-418.

[24] Priyanto S, Mansoori GA, Suwono A. Measurement of property relationships of nano-structure micelles and coacervates of asphaltene in a pure solvent. Chem Eng Sci 2001;56:6933-9.

[25] Sheu EY, Storm DA. Colloidal properties of asphaltenes in organic solvents. In Asphaltenes, Sheu EY, Mullins OC editors: Springer; 1995. p. 1-52.

[26] Sheu EY. Petroleomics and characterization of asphaltene aggregates using small angle scattering. In Asphaltenes, Heavy Oils, and Petroleomics, Mullins OC, Sheu EY, Hammami A, and Marshall AG editors: Springer; 2007. p. 353-74.

[27] Barré L, Simon S, Palermo T. Solution properties of asphaltenes. Langmuir 2008;24:3709-17.

[28] Murgich J, Jesus M, Aray Y. Molecular recognition and molecular mechanics of micelles of some model asphaltenes and resins. Energy Fuels 1996;10:68-76.

[29] Merino-Garcia D, Andersen SI. Calorimetric evidence about the application of the concept of cmc to asphaltene self-association. J Disper Sci Technol 2005;26:217-25.

[30] Buckley JS, Wang J, Creek JL. Solubility of the least-soluble asphaltenes. In Asphaltenes, Heavy Oils, and Petroleomics, Mullins OC, Sheu EY, Hammami A, and Marshall AG editors: Springer; 2007. p. 401-37.

[31] Mullins OC. The Modified Yen Model†. Energy Fuels 2010;24:2179-207.

[32] Pelet R, Behar F, Monin JC. Resins and asphaltenes in the generation and migration of petroleum. Org Geochem 1986;10:481-98.

[33] Koots JA, Speight JG. Relation of petroleum resins to asphaltenes. Fuel 1975;54:179-84.

[34] Loeber L, Muller G, Morel J, Sutton O. Bitumen in colloid science: a chemical, structural and rheological approach. Fuel 1998;77:1443-50.

[35] Andreatta G, Bostrom N, Mullins OC. High-Q ultrasonic determination of the critical nanoaggregate concentration of asphaltenes and the critical micelle concentration of standard surfactants. Langmuir 2005;21:2728-36.

[36] Liu YC, Sheu EY, Chen SH, Storm DA. Fractal structure of asphaltenes in toluene. Fuel 1995;74:13526.

[37] Lisitza NV, Freed DE, Sen PN, Song Y-Q. Study of Asphaltene Nanoaggregation by Nuclear Magnetic Resonance (NMR)†. Energy Fuels 2009;23:1189-93.

[38] da Silva Ramos AC, Haraguchi L, Notrispe FR, Loh W, Mohamed RS. Interfacial and colloidal behavior of asphaltenes obtained from Brazilian crude oils. J Petrol Sci Eng 2001;32:201-16.

[39] Creek JL. Freedom of action in the state of asphaltenes: Escape from conventional wisdom. Energy Fuels 2005;19:1212-24. 
[40] Aguilera-Mercado B, Herdes C, Murgich J, Müller E. Mesoscopic simulation of aggregation of asphaltene and resin molecules in crude oils. Energy Fuels 2006;20:327-38.

[41] Headen TF, Boek ES, Skipper NT. Evidence for Asphaltene Nanoaggregation in Toluene and Heptane from Molecular Dynamics Simulations $\uparrow$. Energy Fuels 2009;23:1220-9.

[42] Headen TF, Boek ES. Molecular Dynamics Simulations of Asphaltene Aggregation in Supercritical Carbon Dioxide with and without Limonene $\dagger$. Energy Fuels 2011;25:503-8.

[43] Antony J, Grimme S. Density functional theory including dispersion corrections for intermolecular interactions in a large benchmark set of biologically relevant molecules. Phys Chem Chem Phys 2006;8:5287-93.

[44] Grimme S. Density functional theory with London dispersion corrections. Wiley Interdiscip Rev Comput Mol Sci 2011;1:211-28.

[45] Alonso M, Woller T, Martín-Martínez FJ, Contreras-García J, Geerlings P, De Proft F. Understanding the Fundamental Role of $\pi / \pi, \sigma / \sigma$, and $\sigma / \pi$ Dispersion Interactions in Shaping Carbon-Based Materials. Chem-Eur J 2014;20:4931-41.

[46] Mackie ID, DiLabio GA. Importance of the Inclusion of Dispersion in the Modeling of Asphaltene Dimers. Energy Fuels 2010;24:6468-75.

[47] Grimme S. Semiempirical GGA-type density functional constructed with a long-range dispersion correction. J Comput Chem 2006;27:1787-99.

[48] Peverati R, Baldridge KK. Implementation and performance of DFT-D with respect to basis set and functional for study of dispersion interactions in nanoscale aromatic hydrocarbons. J Chem Theory Comput 2008;4:2030-48.

[49] Boys SF, Bernardi F. The calculation of small molecular interactions by the differences of separate total energies. Some procedures with reduced errors. Mol Phys 1970;19:553-66.

[50] Schwenke DW, Truhlar DG. Systematic study of basis set superposition errors in the calculated interaction energy of two HF molecules. J Chem Phys 1985;82:2418-26.

[51] Hermann K, Witko M. Binding schemes of adsorbates at metal surfaces: theoretical cluster studies. J Mol Struc-THEOCHEM 1998;458:81-92.

[52] Witko M, Hermann K. Different adsorbate binding mechanisms of hydrocarbons: Theoretical studies for $\mathrm{Cu}(111)-\mathrm{C} 2 \mathrm{H} 2$ and $\mathrm{Cu}(111)-\mathrm{C} 2 \mathrm{H} 4$. Appl Catal A-Gen 1998;172:85-95.

[53] Liu W, Lian JS, Jiang Q. Theoretical study of $\mathrm{C} 2 \mathrm{H} 2$ adsorbed on low-index Cu surfaces. J Phys Chem C 2007;111:18189-94.

[54] Herod AA, Bartle KD, Morgan TJ, Kandiyoti R. Analytical methods for characterizing high-mass complex polydisperse hydrocarbon mixtures: an overview. Chem Rev 2012;112:3892-923.

[55] Wiehe IA. The pendant-core building block model of petroleum residua. Energy Fuels 1994;8:536-44.

[56] Wiehe IA. Asphaltene solubility and fluid compatibility. Energy Fuels 2012;26:4004-16.

[57] Pomerantz AE, Wu Q, Mullins OC, Zare RN. Laser-Based Mass Spectrometric Assessment of Asphaltene Molecular Weight, Molecular Architecture, and Nanoaggregate Number. Energy Fuels 2015, 29: 2833-2842. 
[58] Ruiz-Morales Y, Mullins OC. Polycyclic aromatic hydrocarbons of asphaltenes analyzed by molecular orbital calculations with optical spectroscopy. Energy Fuels 2007;21:256-65.

[59] Gray MR. Consistency of asphaltene chemical structures with pyrolysis and coking behavior. Energy Fuels 2003;17:1566-9.

[60] Dickie JP, Yen TF. Macrostructures of the asphaltic fractions by various instrumental methods. Anal Chem 1967;39:1847-52.

[61] Li DD, Greenfield ML. Chemical compositions of improved model asphalt systems for molecular simulations. Fuel 2014;115:347-56.

[62] Mitra-Kirtley S, Mullins OC, Van Elp J, George SJ, Chen J, Cramer SP. Determination of the nitrogen chemical structures in petroleum asphaltenes using XANES spectroscopy. J Am Chem Soc 1993;115:252-8.

[63] Li DD, Greenfield ML. High internal energies of proposed asphaltene structures. Energy Fuels 2011;25:3698-705.

[64] Zhang L, Greenfield ML. Molecular orientation in model asphalts using molecular simulation. Energy Fuels 2007;21:1102-11.

[65] Martín-Martínez FJ, Fini EH, Buehler MJ. Molecular asphaltene models based on Clar sextet theory. RSC Adv 2015;5:753-9.

[66] Buenrostro-Gonzalez E, Groenzin H, Lira-Galeana C, Mullins OC. The overriding chemical principles that define asphaltenes. Energy Fuels 2001;15:972-8.

[67] McLean JD, Kilpatrick PK. Effects of asphaltene solvency on stability of water-in-crude-oil emulsions. J Colloid Interface Sci 1997;189:242-53.

[68] Murgich J, Abanero JA, Strausz OP. Molecular recognition in aggregates formed by asphaltene and resin molecules from the Athabasca oil sand. Energy Fuels 1999;13:278-86.

[69] Scotti R, Montanari L. Molecular structure and intermolecular interaction of asphaltenes by FT-IR, NMR, EPR. In Structures and Dynamics of Asphaltenes, Mullins OC, Sheu EY editors: Springer; 1998. p. 79-113.

[70] Dennington RD, Keith TA, Millam JM. GaussView, Version 5, Semichem Inc., Shawnee Mission, KS, 2009.

[71] Hunter CA, Sanders JK. The nature of. pi.-. pi. interactions. J Am Chem Soc 1990;112:5525-34.

[72] Pacheco-Sánchez JH, Alvarez-Ramirez F, Martínez-Magadán JM. Morphology of aggregated asphaltene structural models. Energy Fuels 2004;18:1676-86.

[73] Pacheco-Sánchez JH, Zaragoza IP, Martínez-Magadán JM. Asphaltene aggregation under vacuum at different temperatures by molecular dynamics. Energy Fuels 2003;17:1346-55.

[74] Sun H, Rigby D. Polysiloxanes: ab initio force field and structural, conformational and thermophysical properties. Spectrochim Acta A 1997;53:1301-23.

[75] Sun H. COMPASS: an ab initio force-field optimized for condensed-phase applications overview with details on alkane and benzene compounds. J Phys Chem B 1998;102:7338-64.

[76] Carauta AN, Correia JC, Seidl PR, Silva DM. Conformational search and dimerization study of average structures of asphaltenes. J Mol Struc- THEOCHEM 2005;755:1-8. 
[77] Delley B. An all-electron numerical method for solving the local density functional for polyatomic molecules. J Chem Phys 1990;92:508-17.

[78] Delley B. From molecules to solids with the DMol3 approach. J Chem Phys 2000;113:7756-64.

[79] Perdew JP, Burke K, Ernzerhof M. Generalized gradient approximation made simple. Phys Rev Lett 1996;77:3865.

[80] Pollack SS, Yen TF. Structural studies of asphaltics by X-ray small angle scattering. Anal Chem 1970;42:623-9.

[81] Rogel E. Studies on asphaltene aggregation via computational chemistry. Colloid Surface A 1995;104:85-93.

[82] Hunter CA, Lawson KR, Perkins J, Urch CJ. Aromatic interactions. J Chem Soc Perkin Trans 2 2001:651-69.

[83] Evans DJ, Watts RO. On the structure of liquid benzene. Mol Phys 1976;32:93-100.

[84] Narten AH. X-ray diffraction pattern and models of liquid benzene. J Chem Phys 1977;67:2102-8.

[85] Lowden LJ, Chandler D. Theory of intermolecular pair correlations for molecular liquids. Applications to the liquids carbon tetrachloride, carbon disulfide, carbon diselenide, and benzene. J Chem Phys 1974;61:5228-41.

[86] Rodríuez J, Sánchez-Marín J, Torrens F, Ruette F. Molecular aggregation of polycyclic aromatic hydrocarbons. A theoretical modelling of coronene aggregation. J Mol Struc- THEOCHEM 1992;254:42941.

[87] Sedghi M, Goual L, Welch W, Kubelka J. Effect of asphaltene structure on association and aggregation using molecular dynamics. J Phys Chem B 2013;117:5765-76.

[88] Wang P, Dong Z-j, Tan Y-q, Liu Z-y. Investigating the Interactions of the Saturate, Aromatic, Resin, and Asphaltene Four Fractions in Asphalt Binders by Molecular Simulations. Energy Fuels 2015;29:112-21.

[89] Cimino R, Correra S, Del Bianco A, Lockhart TP. Solubility and phase behavior of asphaltenes in hydrocarbon media. In Asphaltenes, Sheu EY, Mullins OC editors: Springer; 1995. p. 97-130.

[90] Alvarez-Ramirez F, Ramirez-Jaramillo E, Ruiz-Morales Y. Calculation of the interaction potential curve between asphaltene-asphaltene, asphaltene-resin, and resin-resin systems using density functional theory. Energy Fuels 2006;20:195-204.

[91] Speight JG. The chemical and physical structure of petroleum: effects on recovery operations. J Petrol Sci Eng 1999;22:3-15.

[92] Schabron JF, Speight JG. The solubility and three-dimensional structure of asphaltenes. Petrol Sci Technol 1998;16:361-75.

[93] Parkash S, Moschopedis SE, Speight JG. Thermal decomposition of resins. Fuel 1980;59:64-6.

[94] Takanohashi T, Sato S, Saito I, Tanaka R. Molecular dynamics simulation of the heat-induced relaxation of asphaltene aggregates. Energy Fuels 2003;17:135-9. 


\section{TOC Graphic}

Micellar structure of asphaltene in a dispersing medium of lighter resins, aromatics, and saturates

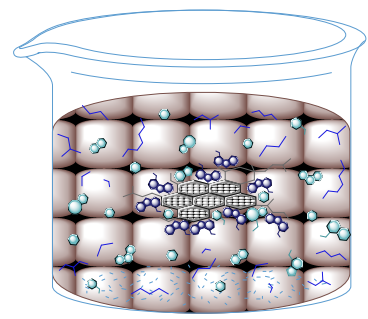

